\title{
A New Modulation Approach for Matrix Converter
}

\author{
S. Pipolo, A.Formentini, A. Trentin \\ P. Zanchetta \\ University of Nottingham \\ Nottingham \\ United Kingdom
}

\author{
M. Calvini, M. Venturini \\ Phase Motion Control s.p.a. \\ Genoa \\ Italy
}

\begin{abstract}
The matrix converter is an AC/AC converter constituted by an array of controlled semiconductor switches that connects directly the three-phase source to the three-phase load. The major development of matrix converters begins when Alesina and Venturini proposed the basic principles of operation in the $80 \mathrm{~s}$. In their modulation methods, through the commonmode-voltage addition technique a voltage ratio of $86.6 \%$ can be obtained. In this paper, a novel method which preserves the same voltage ratio of traditional modulations is proposed. The key concept of this approach is to use only the two closest input voltages instead of all three-phase voltages. The duties cycle are computed on the knowledge of output power demand. With this arrangement, lower switching losses in addition to better harmonic content on output current can be achieved. Hence, this paper presents a detailed mathematical treatment of new modulation approach developed for matrix converter. To validate the proposed strategy, significantly simulation results achieved in co-simulation MATLAB/SIMULINK/PLECS environment will be shown and compared with Venturini's modulation.
\end{abstract}

Index Terms-Matrix converter, Modulation strategy, Losses, Efficiency

\section{SYMBOLS LIST}

* Used to identify reference quantities.

$s_{i j} \quad$ Switch connecting the $i^{\text {th }}$ input phase to the $j^{\text {th }}$ output phase.

$d_{i j} \quad$ Duty cycle of the $s_{i j}$ switch.

$v_{i j} \quad j^{\text {th }}$ input phase voltage.

$v_{o j} j^{t h}$ output phase voltage.

$i_{i j} \quad j^{t h}$ input phase current.

$i_{o j} \quad j^{\text {th }}$ output phase current.

$P_{o} \quad$ Output active power.

$V_{i} \quad$ Input voltage module.

$I_{i} \quad$ Input current module.

$V_{o}$ Output voltage module.

$I_{O} \quad$ Output current module.

$\theta_{i} \quad$ Input voltage phase.

$\theta_{o} \quad$ Output voltage phase.

$\phi_{o} \quad$ Output displacement angle.

$\nu_{o}$ Common-mode output voltage.

$q \quad$ Input-output voltage transfer ratio.

\section{INTRODUCTION}

The matrix converter is usually composed of nine bidirectional switches, where all output phases can be properly linked to each of the three input phases (Fig. 1). Since its introduction in 1976, matrix converter and the subsequent topologies have been used in different applications [1]-[4]. The first rigorous mathematical analysis has been developed by Venturini and Alesina [5], [6].

The first modulation proposed in [6] has a maximum voltage transfer ratio (VTR) of 50\%. To increase it, in [7] and subsequently in [8] the same authors introduce a common-modevoltage (CMV) addition technique, where a voltage ratio of $\frac{\sqrt{3}}{2}$ is obtained. These papers also demonstrate analytically that the voltage ratio of $86.6 \%$ represents an intrinsic limitation of the three-phase to three-phase matrix converter, under balanced supply voltages and balanced output conditions. To have this enhancement in terms of VTR (from 50\% to 86,6\%), the common-mode voltage is computed as a combination of third harmonic grid frequency and third harmonic output frequency. The SVM approach, proposed in [9] achieves the same voltage transfer ratio of $\frac{\sqrt{3}}{2}$ but avoiding to add the third-harmonic components.

In [10], a sub-envelope modulation method applied on a 12switch cell matrix is presented, where the duties cycle are computed taking into account only the closest input voltages.

In this paper, a new common-mode-voltage addition technique is exposed. The proposed approach preserves the same intrinsic limitation about the voltage ratio of $86.6 \%$, but the common-voltage components are computed taking into account the knowledge of output power demand. Comparing with Venturini's method [7], this strategy permits to reduce the commutation losses limiting each output phase switching only between the two closest input phases. In addition, the proposed technique also improves harmonic contents on output currents.

In the next sections, detailed mathematical treatment of new modulation approach including the analytical limitations is presented and discussed.

Finally, to confirm the proposed strategy, significant simulation results achieved in co-simulation MATLAB/SIMULINK/PLECS environment are shown and compared with Venturini's modulation.

\section{Proposed Modulation}

Without loss of generality, in this section the 2 following assumptions are made: 


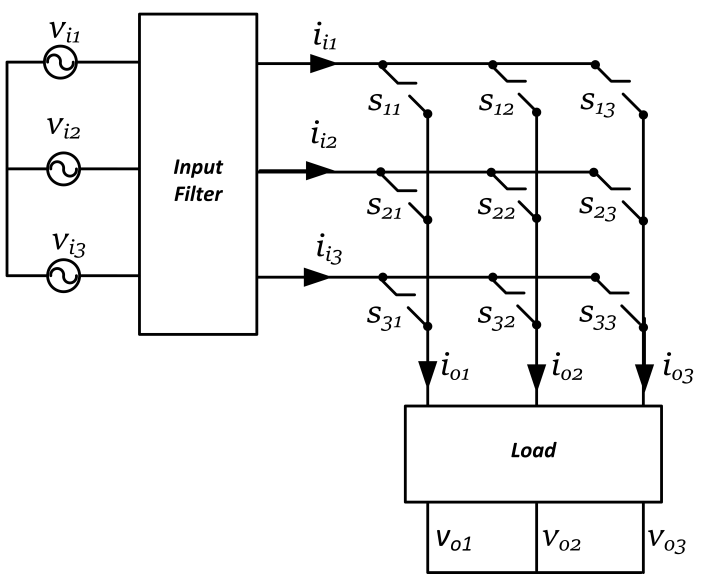

Fig. 1. Matrix converter scheme.

$$
\begin{gathered}
v_{i 1}>v_{i 2}>v_{i 3} \\
v_{o 1}>v_{o 2}>v_{o 3}
\end{gathered}
$$

Let's also assume, at a certain time instant, we have $v_{o 2}^{*}+$ $\nu_{o}<v_{i 2}$. We want to synthesise the desired output voltages connecting the $1^{\text {st }}$ output phase only to the $1^{\text {st }}$ and $2^{\text {nd }}$ input phase. Similarly, the $2^{\text {nd }}$ and $3^{\text {rd }}$ output phases will be connected only to the $2^{\text {nd }}$ and $3^{\text {rd }}$ input phases. Under these assumptions, it is possible to write

$$
v_{o 1}+\nu_{o}=d_{11} v_{i 1}+d_{21} v_{i 2}
$$

Where, with reference to Fig. $1, d_{11}$ refers to switch $s_{11}, d_{21}$ refers to switch $s_{21}$ and so on. Being $d_{11}+d_{21}=1$, it is possible to solve (2) for the duty cycles obtaining

$$
d_{11}=\frac{\left(v_{o 1}^{*}+\nu_{o}-v_{i 2}\right)}{\left(v_{i 1}-v_{i 2}\right)}, \quad d_{21}=\frac{\left(v_{i 1}-v_{o 1}^{*}-\nu_{o}\right)}{\left(v_{i 1}-v_{i 2}\right)}
$$

Analogously,

$$
\begin{aligned}
& v_{o 2}+\nu_{o}=d_{22} v_{i 2}+d_{32} v_{i 3} \\
& v_{o 3}+\nu_{o}=d_{23} v_{i 2}+d_{33} v_{i 3}
\end{aligned}
$$

that results in

$$
\begin{array}{ll}
d_{22}=\frac{\left(v_{o 2}^{*}+\nu_{o}-v_{i 2}\right)}{\left(v_{i 2}-v_{i 3}\right)}, & d_{32}=\frac{\left(v_{i 1}-v_{o 2}^{*}-\nu_{o}\right)}{\left(v_{i 2}-v_{i 3}\right)} \\
d_{23}=\frac{\left(v_{o 3}^{*}+\nu_{o}-v_{i 3}\right)}{\left(v_{i 2}-v_{i 3}\right)}, & d_{33}=\frac{\left(v_{i 2}-v_{o 3}^{*}-\nu_{o}\right)}{\left(v_{i 2}-v_{i 3}\right)}
\end{array}
$$

Similarly to output voltages, input currents can be expressed as function of duty cycles and output currents resulting in

$$
\begin{aligned}
& i_{i 1}=i_{o 1} d_{11} \\
& i_{i 2}=i_{o 1} d_{21}+i_{o 2} d_{22}+i_{o 3} d_{23} \\
& i_{i 3}=i_{o 2} d_{32}+i_{o 3} d_{33}
\end{aligned}
$$

$$
i_{i 1}=\frac{i_{o 1}\left(\nu_{o}+v_{i 2}+v_{o 1}^{*}\right)}{v_{i 1}+v_{i 2}}
$$

In order to ensure sinusoidal input currents, an input current reference can be defined. Equation (7) can then be solved for $\nu_{o}$ resulting in

$$
\nu_{o}^{I}=\frac{i_{i 1}^{*}}{i_{o 1}}\left(v_{i 1}-v_{i 2}\right)+v_{i 2}-v_{o 1}
$$

The input reference current $i_{i 1}^{*}$ is computed imposing a power balance between input and output of matrix converter and assuming a unitary input power factor

$$
i_{i 1}^{*}=\frac{P_{o} v_{i 1}}{V_{i}^{2}}=\frac{\left(i_{o 1} v_{o 1}^{*}+i_{o 2} v_{o 2}^{*}+i_{o 3} v_{o 3}^{*}\right) v_{i 1}}{v_{i 1}^{2}+v_{i 2}^{2}+v_{i 3}^{2}}
$$

If, on the contrary, we have $v_{o 2}^{*}+\nu_{o}>v_{i 2}$ the $1^{\text {st }}$ and $2^{\text {nd }}$ output phases will be only connected to the $1^{\text {st }}$ and $2^{\text {nd }}$ input phases while. the $3^{\text {rd }}$ output phase will be connected only to the $2^{\text {nd }}$ and $3^{\text {rd }}$ input phases. The resulting duty cycles equations become

$$
\begin{array}{ll}
d_{11}=\frac{\left(v_{o 1}^{*}+\nu_{o}-v_{i 2}\right)}{\left(v_{i 1}-v_{i 2}\right)}, & d_{21}=\frac{\left(v_{i 1}-v_{o 1}^{*}-\nu_{o}\right)}{\left(v_{i 1}-v_{i 2}\right)} \\
d_{12}=\frac{\left(v_{o 2}^{*}+\nu_{o}-v_{i 2}\right)}{\left(v_{i 1}-v_{i 2}\right)}, & d_{22}=\frac{\left(v_{i 1}-v_{o 2}^{*}-\nu_{o}\right)}{\left(v_{i 1}-v_{i 2}\right)} \\
d_{23}=\frac{\left(v_{o 3}^{*}+\nu_{o}-v_{i 3}\right)}{\left(v_{i 2}-v_{i 3}\right)}, & d_{33}=\frac{\left(v_{i 2}-v_{o 3}^{*}-\nu_{o}\right)}{\left(v_{i 2}-v_{i 3}\right)}
\end{array}
$$

The input/output currents relations are

$$
\begin{aligned}
& i_{i 1}=i_{o 1} d_{11}+i_{o 2} d_{12} \\
& i_{i 2}=i_{o 1} d_{21}+i_{o 2} d_{22}+i_{o 3} d_{23} \\
& i_{i 3}=i_{o 3} d_{33}
\end{aligned}
$$

To impose sinusoidal input currents, let's substitute $d_{33}$ from (10) in (11c) and solve for $\nu_{o}$

$$
\nu_{o}^{I I}=\frac{i_{i 3}^{*}}{i_{o 3}}\left(v_{i 3}-v_{i 2}\right)+v_{i 2}-v_{o 3}
$$

Also in this case, the input reference current $i_{i 3}^{*}$ is computed from a power balance resulting in (8) and (12) to distinguish the two output common mode values in the two different cases explained.

$$
i_{i 3}^{*}=\frac{P_{o} v_{i 3}}{V_{i}^{2}}=\frac{\left(i_{o 1} v_{o 1}^{*}+i_{o 2} v_{o 2}^{*}+i_{o 3} v_{o 3}^{*}\right) v_{i 3}}{v_{i 1}^{2}+v_{i 2}^{2}+v_{i 3}^{2}}
$$

To summarize, two different common mode voltages must be added to output voltage references to obtain input sinusoidal currents:

$$
\begin{array}{lll}
\nu_{o}=\nu_{o}^{I} & \text { if } & v_{o 2}^{*}+\nu_{o}<v_{i 2} \\
\nu_{o}=\nu_{o}^{I I} & \text { if } & v_{o 2}^{*}+\nu_{o}>v_{i 2}
\end{array}
$$

Substituting (3) in (6a) 


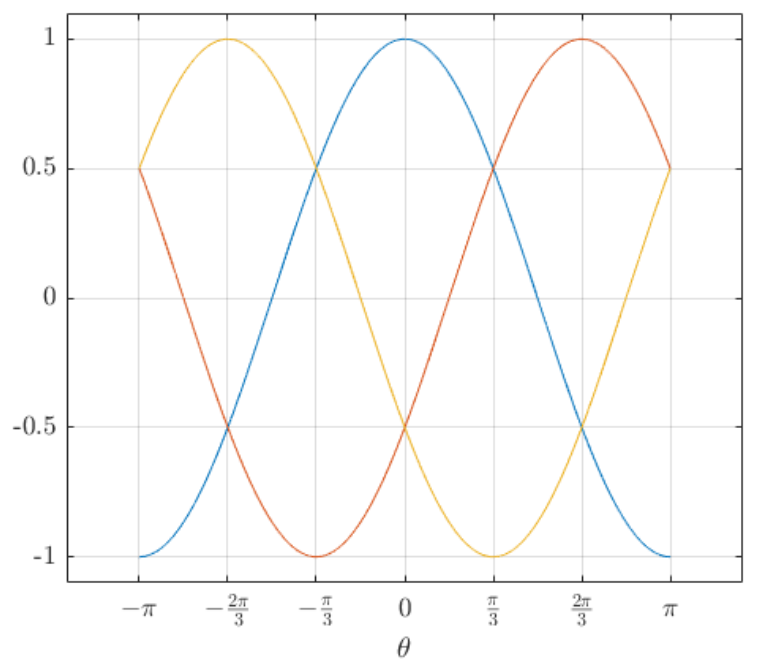

Fig. 2. Normalised three phase system. Blue: $\cos (\theta)$. Red: $\cos \left(\theta-\frac{2 \pi}{3}\right)$. Yellow: $\cos \left(\theta+\frac{2 \pi}{3}\right)$.

\section{MOdUlation OPERATIVE RANGE ANALYSES}

Let's assume that input and output quantities are balanced three phase systems. With reference to Fig. 2, it is possible to observe that the waveforms have a periodicity of $\frac{2 \pi}{3}$. For this reason, the following analysis will be restricted to the interval $\left[-\frac{\pi}{3}, \frac{\pi}{3}\right]$ for both $\theta_{i}$ and $\theta_{o}$. If either $\theta_{i}$ or $\theta_{o}$ are outside the interval, it is sufficient to add or subtract $\frac{2 \pi}{3}$ to the phase until the latter belongs to the mentioned interval.

Let's now do the following assumption

$$
\begin{aligned}
& v_{i 1}=V_{i} \cos \left(\theta_{i}\right) \\
& v_{i 2}=V_{i} \cos \left(\theta_{i}-\frac{2 \pi}{3}\right) \\
& v_{i 3}=V_{i} \cos \left(\theta_{i}+\frac{2 \pi}{3}\right)
\end{aligned}
$$

(15) respects the condition $v_{i 1}>v_{i 2}>v_{i 3}$ only when $0<\theta_{i}<\frac{\pi}{3}$. When $-\frac{\pi}{3}<\theta_{i}<0$, (15) become

$$
\begin{aligned}
& v_{i 1}=V_{i} \cos \left(\theta_{i}\right) \\
& v_{i 2}=V_{i} \cos \left(\theta_{i}+\frac{2 \pi}{3}\right) \\
& v_{i 3}=V_{i} \cos \left(\theta_{i}-\frac{2 \pi}{3}\right)
\end{aligned}
$$

In a similar and more compact form, let's make the following assumptions

$$
\begin{aligned}
& v_{o 1}^{*}=V_{o} \cos \left(\theta_{o}\right) \\
& v_{o 2}^{*}=V_{o} \cos \left(\theta_{o} \mp \frac{2 \pi}{3}\right) \\
& v_{o 3}^{*}=V_{o} \cos \left(\theta_{o} \pm \frac{2 \pi}{3}\right)
\end{aligned}
$$

$$
\begin{aligned}
& i_{o 1}=I_{o} \cos \left(\theta_{o}+\varphi_{o}\right) \\
& i_{o 2}=I_{o} \cos \left(\theta_{o}+\varphi_{o} \mp \frac{2 \pi}{3}\right) \\
& i_{o 3}=i_{o} \cos \left(\theta_{o}+\varphi_{o} \pm \frac{2 \pi}{3}\right)
\end{aligned}
$$

Input current references can be computed with a power balance approach as follow

$$
\begin{gathered}
i_{i 1}^{*}=I_{i}^{*} \cos \left(\theta_{i}\right) \\
i_{i 2}^{*}=I_{i}^{*} \cos \left(\theta_{i} \mp \frac{2 \pi}{3}\right) \\
i_{i 3}^{*}=I_{i}^{*} \cos \left(\theta_{i} \pm \frac{2 \pi}{3}\right) \\
I_{i}^{*} V_{i}=I_{o} V_{o} \cos \left(\varphi_{o}\right) \Rightarrow I_{i}^{*}=\frac{I_{o} V_{o} \cos \left(\varphi_{o}\right)}{V_{i}}
\end{gathered}
$$

Substituting (15-19) in (8) and (12), after some manipulation, equation (21) and (22) are obtained. Please note that to avoid a division by 0 in the latter equations, it must be $\varphi_{o} \in\left[-\frac{\pi}{6}, \frac{\pi}{6}\right]$. This limit will be confirmed in the following analysis.

Equations (21)-(22) represent, in a compact form, the 4 couples of common-mode output voltage for the following four possible cases:

$$
\begin{aligned}
& \theta_{o} \in\left[0, \frac{\pi}{3}\right], \quad \theta_{i} \in\left[0, \frac{\pi}{3}\right] \\
& \theta_{o} \in\left[-\frac{\pi}{3}, 0\right], \quad \theta_{i} \in\left[0, \frac{\pi}{3}\right] \\
& \theta_{o} \in\left[0, \frac{\pi}{3}\right], \quad \theta_{i} \in\left[-\frac{\pi}{3}, 0\right] \\
& \theta_{o} \in\left[-\frac{\pi}{3}, 0\right], \quad \theta_{i} \in\left[-\frac{\pi}{3}, 0\right]
\end{aligned}
$$

\section{A. Maximum voltage transfer ratio}

In this subsection the operative range of the proposed approach will be evaluated, i.e. which voltage transfer ratio can be guaranteed and in which conditions. Also in this case, the analysis will be restricted to the case $\theta_{o} \in\left[0, \frac{\pi}{3}\right], \theta_{i} \in\left[0, \frac{\pi}{3}\right]$ and $v_{o 2}^{*}+\nu_{o}^{I}<v_{i 2}$.

For any value of $\theta_{i}, \theta_{o}$ and $\varphi_{o}$ the output voltage references must be between the maximum and the minimum input voltages, i.e. the following relations must hold

$$
\begin{aligned}
& v_{o 1}^{*}+\nu_{o}^{I}<v_{i 1} \\
& v_{o 3}^{*}+\nu_{o}^{I}>v_{i 3}
\end{aligned}
$$

Substituting (15a), (17a) and (21) in (25) and rearranging terms

$$
\begin{gathered}
\frac{\sigma_{2}\left(V_{i} \cos \left(\varphi_{o}+\theta_{o}\right)+V_{o} \cos \left(\varphi_{o}\right) \cos \left(\theta_{i}\right)\right)}{\sigma_{3}}<0 \\
\sigma_{2}=\sqrt{3} \cos \left(\theta_{i}+\frac{\pi}{3}\right) \\
\sigma_{3}=\cos \left(\varphi_{o}+\theta_{o}\right)
\end{gathered}
$$

since $\sigma_{2}$ and $\sigma_{3}$ are always positive in the analysis interval, (27) can be further rewritten as 


$$
\begin{gathered}
\nu_{o}^{I}=\left(\frac{\cos \left(\varphi_{o}\right) \cos \left(\theta_{i}\right)\left(\cos \left(\theta_{i}\right)-\cos \left(\theta_{i} \mp \frac{2 \pi}{3}\right)\right)}{\cos \left(\theta_{o}+\varphi_{o}\right)}-\cos \left(\theta_{o}\right)\right) V_{o}+\cos \left(\theta_{i} \mp \frac{2 \pi}{3}\right) V_{i} \\
\nu_{o}^{I I}=\left(\frac{\cos \left(\varphi_{o}\right) \cos \left(\theta_{i} \pm \frac{2 \pi}{3}\right)\left(\cos \left(\theta_{i} \pm \frac{2 \pi}{3}\right)-\cos \left(\theta_{i} \mp \frac{2 \pi}{3}\right)\right)}{\cos \left(\theta_{o} \pm \frac{2 \pi}{3}+\varphi_{o}\right)}-\cos \left(\theta_{o} \pm \frac{2 \pi}{3}\right)\right) V_{o}+\cos \left(\theta_{i} \mp \frac{2 \pi}{3}\right) V_{i} \\
\sigma_{1}=\left(\cos \left(2 \theta_{i}-\varphi_{o}+\frac{\pi}{6}\right)+\cos \left(2 \theta_{i}+\varphi_{o}+\frac{\pi}{6}\right)-2 \cos \left(2 \theta_{o}+\varphi_{o}+\frac{\pi}{6}\right)-\sin \left(\varphi_{o}\right)\right)
\end{gathered}
$$

$$
q \triangleq \frac{V_{o}}{V_{i}}<\frac{\cos \left(\varphi_{o}+\theta_{o}\right)}{\cos \left(\varphi_{o}\right) \cos \left(\theta_{i}\right)}
$$

Equation (28) provides an analytical expression of the maximum value of $q$ as function of $\theta_{i}$ and $\theta_{o}$. The maximum voltage transfer ratio $q_{\max }$ can be found numerically solving the following programming problem:

$$
\begin{aligned}
\min _{\theta_{i}, \theta_{o}} & \frac{\cos \left(\varphi_{o}+\theta_{o}\right)}{\cos \left(\varphi_{o}\right) \cos \left(\theta_{i}\right)} \\
\text { s.t. } & \sigma_{1}<0 \\
& 0<\theta_{i}<\frac{\pi}{3} \\
& 0<\theta_{o}<\frac{\pi}{3}
\end{aligned}
$$

Constraint (29b) ensures condition $v_{o 2}^{*}+\nu_{o}^{I}<v_{i 2}$ is satisfied ( $\sigma_{1}$ from (23)). If the same steps are applied to (25) a similar programming problem is obtained. The maximum voltage transfer ratio can then be evaluated numerically for different values of $\varphi_{o} \in\left[-\frac{\pi}{6}, \frac{\pi}{6}\right]$. At each step, the more restrictive value returned by the two programming problems is used. The procedure permits to create the modulation operative range reported in Fig. 3. As can be noted, the nominal matrix converter voltage transfer ratio of $\frac{\sqrt{3}}{2}$ can be obtained for the output power factor very close to 1 . When the power factor decrease, the maximum voltage transfer ratio must decrease accordingly.

The analysis performed till now has been carried out assuming the case of (14a) and (24a). The presented results have been however verified for all possible cases adopting the some described procedure and the same outcomes have been obtained.

\section{Simulation Results}

The proposed approach has been verified in the cosimulation MATLAB/SIMULINK/PLECS environment with a $500 \mathrm{~kW}$ matrix converter which fed an ohmic-inductive load, where resistance value is $0.24 \Omega$, the inductance value is $100 \mu \mathrm{H}$ and the output angular frequency is $550 \mathrm{rad} / \mathrm{s}$; thus the power factor is set to 0.97 , which corresponds to a $\varphi_{o}=0.2253 \mathrm{rad}$, less than $\left|\varphi_{o}\right|=\frac{\pi}{40}$. In order to highlight the behaviour of the proposed technique in worst-case scenario, the voltage transfer ratio is fixed to the maximum value of $\frac{\sqrt{3}}{2}$. As introduced in the previous section, this operative point corresponds to a region, which is located outside the

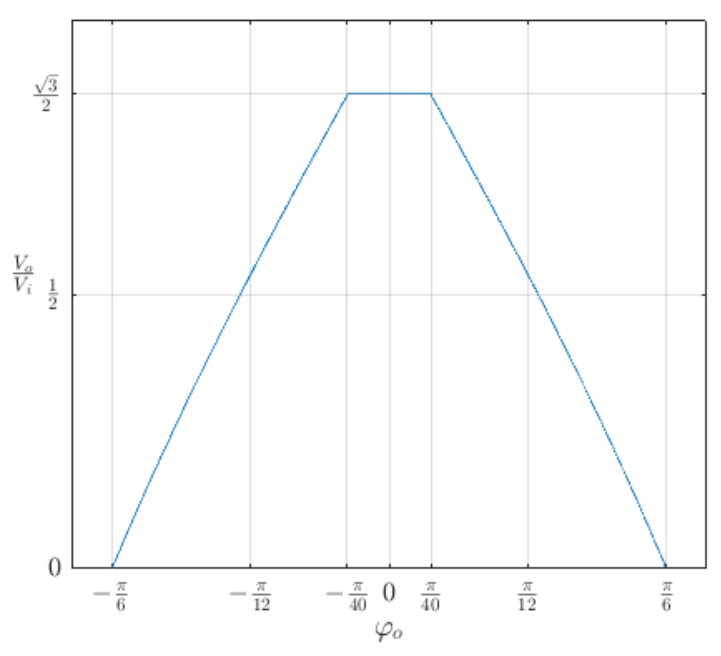

Fig. 3. Maximum voltage transfer ratio in function of $\varphi_{o}$.

achievable operative conditions (Fig. 3). This means that the proposed method cannot guarantee the proper operation at this set point. However, in order to preserve its benefits whenever the operative conditions fall outside the achievable operative conditions ones, a hybrid modulation can be applied.

The hybrid modulation consists in using proposed modulation assisted by Venturini's modulation. This can be easily implemented by monitoring the common mode voltage and voltage reference. In particular, whenever the modulation is running and applied to the converter, it is necessary to switch to Venturini's modulation if the sum of the common mode voltage and the voltage reference (computed with the proposed method) exceeds the maximum (or the minimum) input voltage.

The converter is equipped with SEMIKRON custom bidirectional switches modules composed by IGC193T120T8RM Infineon's chip. To manage the transitions of the non-ideal switches and thus, to consider the actual behaviour of the converter, four-step current commutation [11] is implemented taking into account a dead time equal to $1 \mu \mathrm{s}$ between the steps. Input filter depicted in Fig. 4, is designed according to [12] considering the parameters listed in Table I.

All the comparisons are performed paralleling the hybrid modulation with Venturini's method for equal THD on the input current. In order to achieve this, the switching frequencies are established to $10 \mathrm{kHz}$ for hybrid technique and to $7.1 \mathrm{kHz}$ 
for Venturini's method. With this arrangement, the THD on the input current is fixed to $8.48 \%$ of the fundamental frequency for both modulations.

TABLE I

INPUT FILTER PARAMETERS

\begin{tabular}{c|c}
\hline Main inductance, $\mathbf{L}_{\text {main }}$ & $5 \mu \mathbf{H}$ \\
Grid inductance, $\mathbf{L}_{\text {grid }}$ & $15 \mu \mathbf{H}$ \\
Series grid resistance, $\mathbf{R}_{\text {grid }}$ & $\mathbf{4 0} \mathbf{~} \Omega$ \\
Damping resistor, $\mathbf{R}_{\text {damp }}$ & $\mathbf{5 0 0} \mathbf{~ m} \Omega$ \\
Star-connected capacitors, $\mathbf{C}$ & $\mathbf{3 0 0} \mu \mathbf{F}$ \\
\hline
\end{tabular}

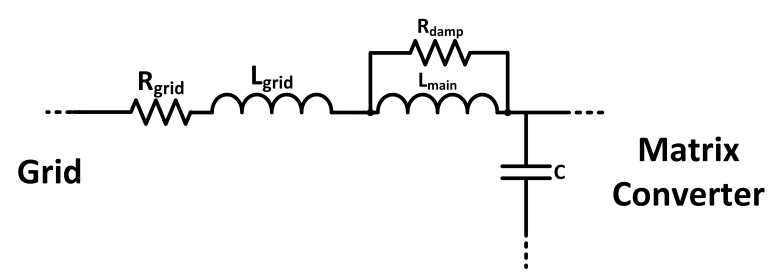

Fig. 4. Input filter used in simulation analysis

In Fig. 5, the input and output currents achieved with the hybrid method are shown, where the input ones are measured on grid-side. By analysing the harmonics spectrum of the input currents achieved with the new approach, shown in Fig. 8, it can be noted that the relevant contribution is located at the cutoff frequency of the input filter. The highest harmonic introduced by new modulation technique is located at $5^{\text {th }}$ harmonic of the fundamental frequency, likewise to Venturini's method (Fig. 6). As usual for matrix converter, a low harmonic content can be obtained on the output currents, as demonstrate by FFTs depicted in Fig. 7 and in Fig. 9. However, a lower THD of the $2.27 \%$ on output currents is obtained with the hybrid method against $2.55 \%$ achieved with Venturini's method Fig. 7.

In Fig. 10 and Fig. 11 output voltage of only one output phase achieved with Venturini's method and hybrid method is shown respectively. According to the main idea of proposed modulation, it can be noted that voltage switched range for the hybrid method is very contained compared with Venturini's one.

In Fig. 12 the conduction and switching losses are compared, where it can be noted that the hybrid technique obtains lower switching losses. The new approach attains a $3.5 \mathrm{~kW}$ of switching losses versus $5.8 \mathrm{~kW}$ of traditional modulation. This corresponds to an improvement of $0.4 \%$ referred to the rated power of $500 \mathrm{~kW}(0.7 \%$ vs. $1.1 \%)$. Since the current which feeds load is the same for both techniques, the conduction losses are quite similar for both approaches. On the other hand, for both modulation strategies, always occur that one IGBT (MOSFET) and one diode are conducting simultaneously. On the contrary, since the proposed approach acts on the closest input voltages rather than of using all three phases voltages Fig. 10 and Fig. 11, the switching losses are significantly reduced.
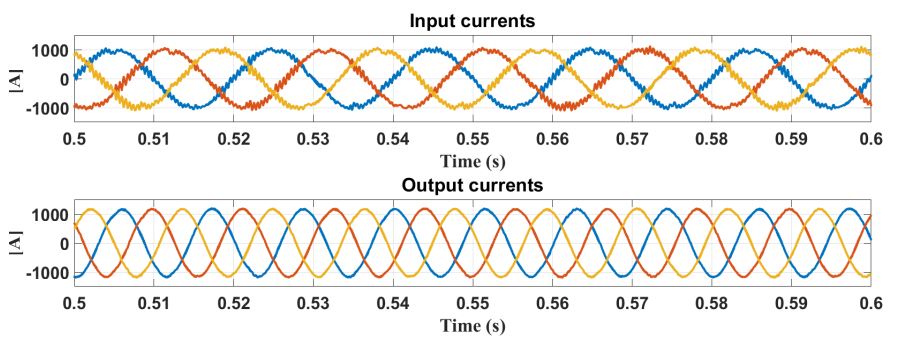

Fig. 5. Filtered input and output currents waveforms achieved with hybrid method

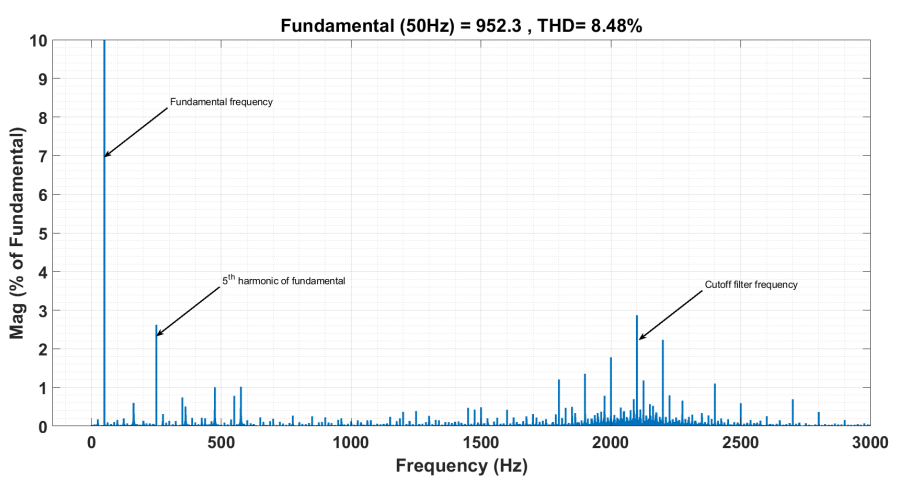

Fig. 6. Harmonics Spectrum of input current achieved with Venturini's method

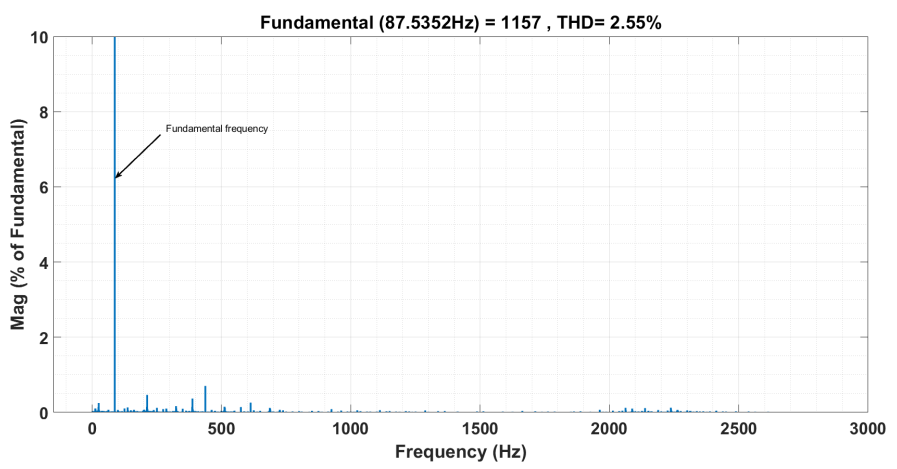

Fig. 7. Harmonics Spectrum of output current achieved with Venturini's method

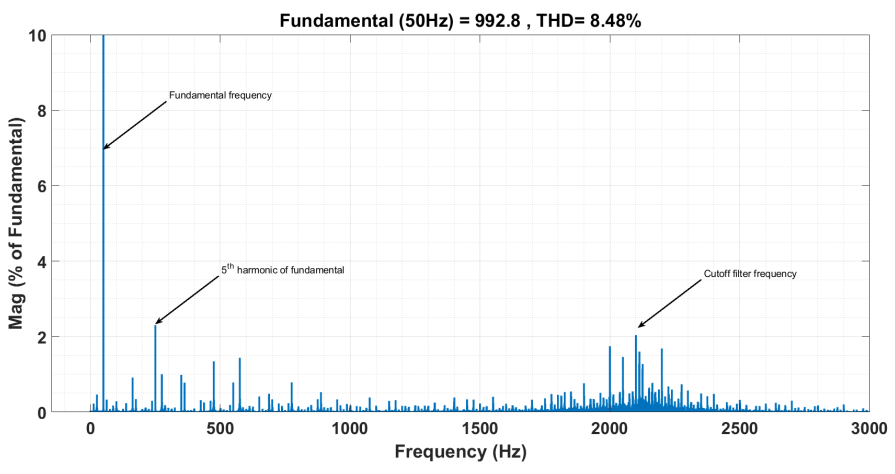

Fig. 8. Harmonics Spectrum of input current achieved with hybrid method 


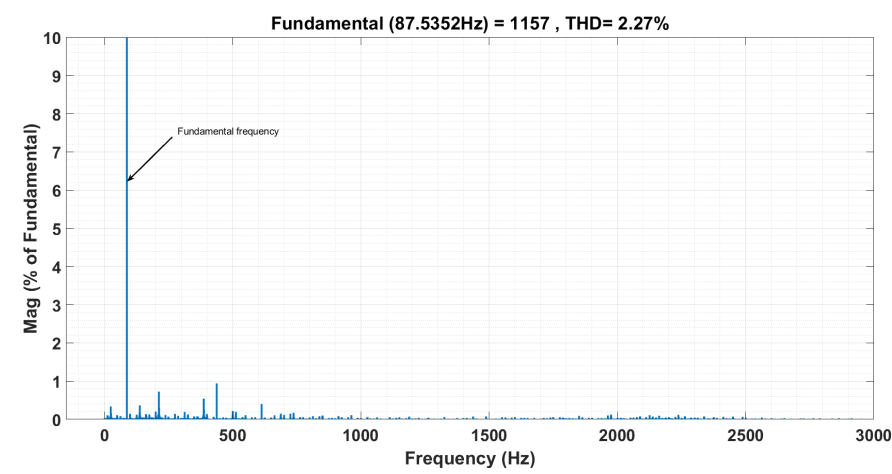

Fig. 9. Harmonics Spectrum of output current achieved with hybrid method

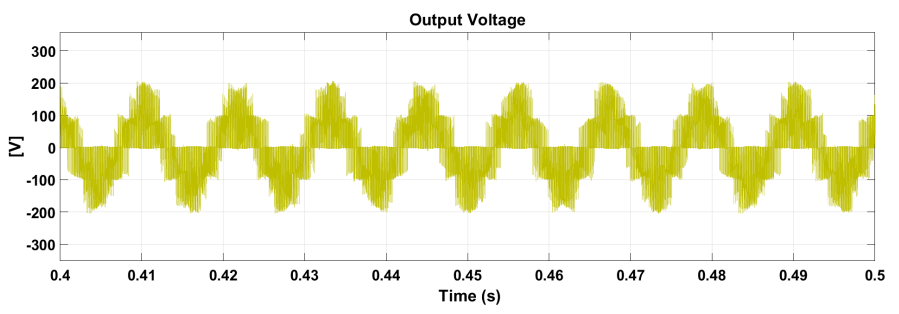

Fig. 10. Output voltage achieved with Venturini's method

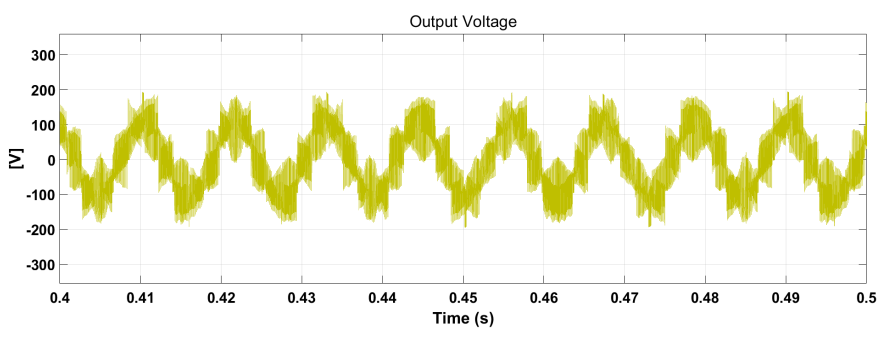

Fig. 11. Output voltage achieved with hybrid method

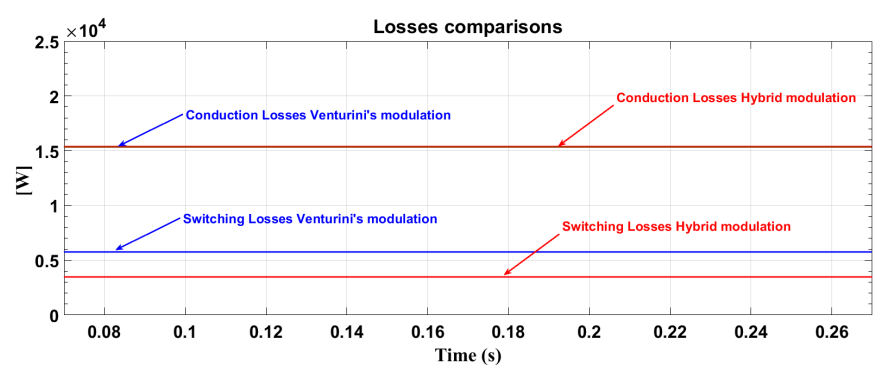

Fig. 12. Conduction and switching losses achieved with Venturini's method (blue) and hybrid method (red)

As shown in Section II, all the operative cases corresponding to a $\left|\varphi_{o}\right|<\frac{\pi}{40}$ with voltage ratio set to $\frac{\sqrt{3}}{2}$, imply to use a proposed technique without the support of Venturini's modulation. In those cases, compared with considered set-up, it will always have a greater losses reduction (CMV will be only computed across two closest input voltages) and consequently a lower harmonic content on output currents.

\section{CONClusions}

A new modulation approach designed for matrix converter has been introduced and validated in this paper. The mathematical treatment has shown how the proposed approach computed common-mode-voltage in a different way than the traditional modulation strategy. In particular, the proposed approach uses the closest input voltages instead of using all three phases voltages like traditional Venturini's method. Through this enhancement, the switching losses can be significantly reduced, as well as the THD on output current decreases accordingly. Such a method permits to preserve the usual intrinsic voltage transfer ratio of $\frac{\sqrt{3}}{2}$ when a power factor close to unity is required (more than $\cos \left(\frac{\pi}{40}\right)$ ). Alternatively, if a smaller power factor is required, in order to attain the benefits of proposed strategy with the maximum voltage ratio, it is possible to apply a hybrid modulation by supporting proposed approach with traditional Venturini's modulation. In order to validate the hybrid technique, significantly simulations have been carried out in co-simulation MATLAB/SIMULINK/PLECS environment. Simulation results, performed for equal THD on the input current, have shown that compared with traditional Venturini's modulation, the novel method achieves lower switching losses and a lower harmonic content in output current.

\section{REFERENCES}

[1] A. Formentini, L. De Lillo, M. Marchesoni, A. Trentin, P. Wheeler, and P. Zanchetta, "A new mains voltage observer for PMSM drives fed by matrix converters," in 16th European Conference on Power Electronics and Applications, 2014, pp. 1-10.

[2] L. Empringham, J. W. Kolar, J. Rodriguez, P. W. Wheeler, and J. C. Clare, "Technological issues and industrial application of matrix converters: A review," IEEE Transactions on Industrial Electronics, vol. 60, no. 10 , pp. $4260-4271,2013$.

[3] S. Pipolo, S. Bifaretti, A. Lidozzi, L. Solero, F. Crescimbini, and P. Zanchetta, "The ROMAtrix converter: Concept and operation," in 2017 IEEE Southern Power Electronics Conference (SPEC). IEEE, 2017, pp. 1-6.

[4] A. Shahani, K. Basu, and N. Mohan, "A power electronic transformer based on indirect matrix converter for pwm ac drive with lossless commutation of leakage energy," 2012.

[5] A. Alesina and M. Venturini, "The generalized transformer: a new bidirectional sinusoidal waveform frequency converter with continuous variable adjustable input power factor," IEEE PESC'80, pp. 242-252, 1980.

[6] M. Venturini, "A new sine wave in sine wave out, conversion technique which eliminates reactive elements," Proc. Powercon 7, 1980.

[7] A. Alesina and M. Venturini, "Intrinsic amplitude limits and optimum design of 9-switches direct pwm ac-ac converters," in PESC'88 Record., 19th Annual IEEE Power Electronics Specialists Conference. IEEE, 1988, pp. 1284-1291.

[8] A. Alesina and M. G. Venturini, "Analysis and design of optimumamplitude nine-switch direct ac-ac converters," IEEE Transactions on Power Electronics, vol. 4, no. 1, pp. 101-112, 1989.

[9] L. Huber and D. Borojevic, "Space vector modulator for forced commutated cycloconverters," in Conference Record of the IEEE Industry Applications Society Annual Meeting,. IEEE, 1989, pp. 871-876.

[10] F. L. Luo and Z. Y. Pan, "Sub-envelope modulation method to reduce total harmonic distortion of ac/ac matrix converters," IEE ProceedingsElectric Power Applications, vol. 153, no. 6, pp. 856-863, 2006.

[11] N. Burany, "Safe control of four-quadrant switches," in Conference Record of the IEEE Industry Applications Society Annual Meeting,. IEEE, 1989, pp. 1190-1194.

[12] A. Trentin, P. Zanchetta, J. Clare, and P. Wheeler, "Automated optimal design of input filters for direct ac/ac matrix converters," IEEE Transactions on Industrial Electronics, vol. 59, no. 7, pp. 2811-2823, 2012. 